\title{
CONTROLE DE PLANTAS DANINHAS NA CULTURA DO MILHO (Zea mays L.)
POR MEIO DE HERBICIDAS
}

\section{C.A.L. DOS SANTOS* e A. ROZANSKI*}

Pesquisadores Científicos do Instituto Biológico, Seção de Herbicidas. Caixa Postal 70, 13.100 Campinas.

Recebido para publicação em 16.11.79.

\section{RESUMO}

Com o objetivo de se verificar a ação do butylate, aplicado isoladamente e em mistura com atrazine, no controle de plantas daninhas da cultura do milho, foi instalado um experimento de campo em solo fino areno-argiloso.

Foram utilizados os seguintes tratamentos: butylate a 2,$80 ; 3,60$ e $4,32 \mathrm{~kg} / \mathrm{ha}$ (p.p.i.); butylate + atrazine a $3,24+0,80 ; 3,24+1,20$ e $3,60+0,96$ $\mathrm{kg} / \mathrm{ha}$ (p.p.i.); atrazine a $3,00 \mathrm{~kg} / \mathrm{ha}$ e atrazine + metolachlor a $1,40+2,10 \mathrm{~kg} / \mathrm{ha}$, ambos aplicados em pré-emergência e empregados como herbicidas padrão para a cultura.

As plantas daninhas encontradas foram: tiririca - Cyperus rotundus L., carurú comum .:maranthus viridis L., capim de colchão - Digitaria sanguinalis (L.) Scop. e capim pé-de-galinha Eleusine indica (L.) Gaertn.

Butylate nas três doses apresentou-se bem contra C. rotundus e E. indica; nas doses de 3,60 e $4,32 \mathrm{~kg}$ foram obtidos bons resultados sobre $D$. sanguinalis. Butylate + atrazine controlou, nas três doses, todas as espécies incidentes, o mesmo ocorrendo com a mistura atrazine + metolachlor. Atrazine foi mais eficiente para A. viridis $e E$. indica.

Nas condições em que foi conduzido o experimento nenhum dos herbicidas foi prejudicial para a cultura.

UNITERMOS: milho, plantas daninhas, atrazine + butylate.

\section{SUMMARY}

WEED CONTROL IN MAIZE (Zea mays L.) WITH HERBICIDES.

Butylate at $2.80 ; 3.60$ and $4.32 \mathrm{~kg} / \mathrm{ha}$ and butylate + atrazine at. $3.24+0.80 ; 3.24+1.20$ and 3.60 $+0.96 \mathrm{~kg}$, were applied in preplant incorporated; atrazine at $3.00 \mathrm{~kg}$ and atrazine + metolachlor at $1.40+2.10 \mathrm{~kg}$ were applied in preemergence on corn.

The weeds were represented by Cyperus rotundus L., Amaranthus viridis L., Digitaria sanguinalis (L.) Scop. and Eleusine indica (L.) Gaertn.

Butylate + atrazine, in all rates, atrazine + metolachlor and atrazine gave good control of the weeds in general. Butylate, in the three rates, controlled C. rotundus and E. indica; at 3.60 and $4.32 \mathrm{~kg} / \mathrm{ha}$ controlled well D. sanguinalis.

The herbicides did not cause injuries to the crop. KEYWORDS: Zea mays, weeds, atrazine +
butylate.

\section{INTRODUÇÃO}

O controle das plantas daninhas na cultura do milho é de grande importância para aqueles que desejam obter um alto rendimento, tornando-se necessário eliminá-las na época oportuna e por processos adequados.

O emprego de herbicidas é uma prática que visa combater o mato das culturas, especialmente na linha de plantio, de maneira mais racional, salientando-se que o uso de misturas de herbicidas permite um controle de maior número de espécies de plantas daninhas, razão pela qual essa técnica vem se tornando mais acentuada, conforme trabalhos publicados (2 e 6).

No experimento aqui relatado foi estudado o butylate, que tem sido citado como excelente herbicida para milho (3,10 a 11); aplicado isoladamente e em mistura com atrazine, comparandose com atrazine e atrazine + metolachlor, os quais têm apresentado excelentes resultados como atestam vários trabalhos técnicos $(1,4,13$ e 15).

\section{MATERIAIS E MÉTODOS}

Um experimento de campo foi instalado no município de Araras-SP, em novembro de 1978, em solo fino areno argiloso e com as seguintes características: $36,7 \%$ de argila, $10,0 \%$ de limo, 
$40,4 \%$ de areia fina e $12,9 \%$ de areia grossa: $\mathrm{pH}$ de 5,92 e $2,8 \%$ de matéria orgânica.

$\mathrm{O}$ delineamento experimental escolhido foi o de blocos ao acaso com nove tratamentos e quatro repetições, para possibilitar a análise estatística do «stand» e da produção do milho em palha e em grão. Cada parcela tinha uma área de 17,00 $\mathrm{m}^{2}$, abrangendo quatro linhas espaçadas de $0,85 \mathrm{~m}$ e com $5,00 \mathrm{~m}$ de comprimento.

Os tratamentos utilizados foram os seguintes: butylate a $2,80-3,60$ e 4,32 kg/ha; butylate + atrazine a $3,24+0,80 ; 3,24+1,20$ e $3,60+0,96 \mathrm{~kg} / \mathrm{ha}$; atrazine a $3,00 \mathrm{~kg} / \mathrm{ha}$ e atrazine + metolachlor a $1,40+2,10 \mathrm{~kg} / \mathrm{ha}$. Além desses tratamentos foi incluída uma testemunha, mantida no limpo mediante capina com enxada.

As pulverizações foram realizadas no mesmo dia do plantio, sendo que butylate, quer utilizado isoladamente ou em mistura com atrazine, foi aplicado em pré-plantio e incorporado imediatamente ao solo com enxada rotativa a uma profundidade de $8 \mathrm{~cm}$. Os tratamentos atrazine e atrazine + metolachlor foram feitos logo após o plantio.

Um pulverizador costal com bico leque $n .{ }^{\circ}$ 80.03, foi o aparelho empregado para aplicação dos herbicidas, sendo que $5001 /$ ha o gasto de calda. A temperatura era de $23^{\circ} \mathrm{C}$ à sombra e com vento fraco.

O plantio foi realizado com plantadeira a tração animal, em terreno plano e bem preparado, a uma profundidade de $6 \mathrm{~cm}$ e com um gasto de 11 sementes por metro linear. A variedade utilizada foi a H-7974 da Secretaria da Agricultura do Estado de São Paulo. A adubação empregada foi a constituída pela fórmula de 8-12-20, com 400 $\mathrm{kg} / \mathrm{ha}$ da mistura.

A eficiência dos tratamentos foi verificada pela contagem das plantas daninhas, 30 dias após a aplicação dos produtos, em uma área de $0,50 \mathrm{~m}^{2}$ $(0,50 \times 1,00 \mathrm{~m})$, representativa da infestação média de cada parcela. O cálculo dos valores representativos dessa eficiência foi feito com base na diferença entre o número de ervas da testemunha e das parcelas tratadas, transformada em porcentagem.

As plantas daninhas encontradas no experimento foram: Cyperus rotundus L., Amaranthus viridis L., Digitaria sanguinalis (L.) Scop e Eleusine indica (L.) Gaertn.

$\mathrm{O}$ efeito dos herbicidas sobre a cultura também foi estudado, observando-se o desenvolvimento do milho desde a germinação até a colheita, realizada em maio de 1979. A contagem do «stand» e a colheita foram feitas nas duas linhas centrais de cada parcela.

\section{RESULTADOS E DISCUSSÃO}

No quadro 1 encontram-se os dados obtidos pela ação dos herbicidas sobre as plantas daninhas e cujos resultados são os seguintes:

Cyperus rotundus: teve um ótimo controle por parte dos tratamentos, com exceção para atrazine que apresentou de regular a bom.

Amaranthus viridis: atrazine, atrazine + metolachlor e butylate + atrazine (nas 3 doses) proporcionaram excelentes resultados sobre essa espécie; butylate aplicado isoladamente não foi eficiente, mesmo na dose maior.

Digitaria sanguinalis: controle excelente por parte de atrazine + metolachlor, seguido por butylate a 4,32 kg e butylate + atrazine (nas 3 doses). Butylate a 3,60 kg evidenciou uma ação considerada boa, sendo que a dose menor não foi eficiente. Atrazine apresentouse regularmente.

Quadro 1 - Controle de plantas daninhas aos 30 dias após as pulverizaçōes na cultura do milho, em Araras-SP. Tratamento: 20 de novembro de 1978. Contagem das plantas daninhas: 19 de dezembro de 1978 .

\begin{tabular}{lcccccc}
\hline Tratamentos & $\begin{array}{c}\text { Ingredien- } \\
\text { te ativo }\end{array}$ & $\begin{array}{c}\text { Cyperus } \\
\text { rotundus }\end{array}$ & $\begin{array}{c}\text { Amaranthus } \\
\text { viridis }\end{array}$ & $\begin{array}{c}\text { Digitaria } \\
\text { sanguinalis }\end{array}$ & $\begin{array}{c}\text { Eleusine } \\
\text { indica }\end{array}$ & Controle geral \\
\hline \multirow{3}{*}{$\begin{array}{l}\text { butylate } \\
\mathrm{kg} / \mathrm{ha}\end{array}$} & $\%$ & $\%$ & $\%$ & $\%$ & $\%$ \\
butylate & 2,80 & 98,0 & 55,1 & 75,4 & 87,5 & 71,3 \\
butylate & 3,60 & 99,0 & 60,7 & 86,8 & 93,8 & 76,7 \\
\hline butylate + atrazine & 4,32 & 100,0 & 74,1 & 91,8 & 100,0 & 85,3 \\
butylate + atrazine & $3,24+0,80$ & 100,0 & 93,1 & 90,1 & 100,0 & 95,0 \\
butylate + atrazine & $3,24+1,20$ & 100,0 & 93,1 & 91,8 & 100,0 & 95,2 \\
\hline atrazine & $3,60+0,96$ & 100,0 & 94,8 & 91,8 & 100,0 & 96,1 \\
\hline atrazine + metolachlor & $1,40+2,10$ & 100,0 & 99,1 & 100,0 & 100,0 & 99,5 \\
\hline Testemunha (número de plantas daninhas $/ \mathrm{m}^{2}$ ) & 111 & 101 & 75 & 34 & 321 \\
\hline
\end{tabular}


Quadro 2 - Contagem das plantas e produção de milho em palha e em grão, nas 2 linhas centrais de cada parcela, em Araras, SP. Contagem das plantas de milho: 19 de dezembro de 1978 . Colheita: 11 de maio de 1979.

\begin{tabular}{lcccc}
\hline \multirow{2}{*}{ Tratamentos } & $\begin{array}{c}\text { Ingrediente } \\
\text { ativo } \\
\text { (kg/ha) }\end{array}$ & $\begin{array}{l}\text { Número de } \\
\text { plantas de } \\
\text { milho (4 re- } \\
\text { tiçöes) }\end{array}$ & Palha (kg/ha) & Grão (kg/ha) \\
\cline { 4 - 5 } $\begin{array}{l}\text { butylate } \\
\text { butylate } \\
\text { butylate }\end{array}$ & 2,80 & 208 & 4.800 & 3.250 \\
\hline $\begin{array}{l}\text { butylate + atrazine } \\
\text { butylate + atrazine } \\
\text { butylate + atrazine }\end{array}$ & 3,60 & 205 & 4.600 & 3.200 \\
\hline atrazine & 4,32 & 205 & 4.750 & 3.375 \\
\hline atrazine & $3,24+0,80$ & 199 & 4.550 & 3.025 \\
\hline Testemunha & $3,60+0,96$ & 201 & 4.750 & 3.125 \\
\hline C.V. \% & 3,00 & 201 & 4.600 & 3.250 \\
\hline F & $1,50+2,10$ & 202 & 4.825 & 3.250 \\
\hline
\end{tabular}

Eleusine indica: os melhores resultados foram obtidos com butylate a $4,32 \mathrm{~kg}$, butylate + atrazine (nas 3 doses) e atrazine + metolachlor todos com $100 \%$ de controle, vindo a seguir atrazine e butylate a 3,60 e $2,80 \mathrm{~kg}$.

Com relação ao controle geral verifica-se que os melhores índices foram obtidos com atrazine + metolachlor, seguido por butylate + atrazine (nas 3 doses) e atrazine. Butylate apresentou-se melhor a $4,32 \mathrm{~kg}$, sendo que os demais tratamentos não foram eficientes.

Os resultados apresentados por butylate estão de acordo com os trabalhos de vários pesquisadores, entre os quais Uriarte (14), que verificou a ótima ação desse herbicida para $C$. rotundus; Hood (7) cita que butylate a 3,24 - 3,60 $\mathrm{kg} / \mathrm{ha}$ é recomendado para o controle de $C$. rotundus e também de outras monocotiledôneas; Horowitz (8) observou que o referido herbicida apresentou resultado satisfatório no combate de $C$. rotundus, mas não foi eficiente para dicotiledôneas.

Com relação a mistura butylate + atrazine, foi verificada maior ação sobre as ervas, tanto monocotiledôneas como dicotiledôneas, coincidindo com os trabalhos de Chandra Singh (1), Sarpe (12) e Veselovskii (15), os quais indicam um maior controle sobre as espécies daninhas pertencentes a esses dois grupos botânicos. Crespy (5) diz que obteve bom resultado utilizando $3,96 \mathrm{~kg}$ de butylate e $1,00 \mathrm{~kg}$ de atrazine por hectare.

O bom comportamento de atrazine + metolachlor observado no experimento, está em concordância com o verificado por Salto (9), o qual observou uma boa eficiência contra $C$. rotundus e D. sanguinalis, salientando que o emprego dessa mistura reduziu grandemente o risco de injúria para culturas seguintes.

Nas condições em que foi realizado o experimento não se observou nenhum sinal de fitotoxicidade para o milho por parte dos tratamentos, bem como a produção, tanto em palha como em grão, não foi prejudicada. A análise estatística, pelo método da variância, dos dados de produção não revelou diferenças significativas entre os tratamentos. Os coeficientes de variação foram de $3,26 \%$ para o «stand» e $9,85 \%$ e $8,46 \%$, respectivamente para os pesos em palha e em grão. 


\section{LITERATURA CITADA}

1. Chandra Singh, D.J. e Narayana Rao, K. Herbicidal control of weeds in maize. Pesticides (1975) 9(10): 19-21. Agric. Coll. Andhra Pradesh Agric. Univ. Bapatla, India. From abstracts on Tropical Agriculture 2,08773. (Weed Abstract, 26(11): 345, 1977).

2. Chandra Singh, D.J. e Narayana Rao, K. Herbicidal control of weeds in maize. Indiam Journal of weed Science (1974) 6(1): 711. Andhra Pradesh Agric. Univ. Agric. Coll. Bapatla, India (Weed Abstract 26(11): 345, 1977.

3. Coelho, J.P. e Hostalácio, S. Competição de herbicidas no controle de ervas daninhas na cultura do milho (Zea mays L.). In: Sem. Bras. Herb. Ervas Dan. XI. Londrina, 1976. Resumos, 46.

4. Covolo, L. e Stefaelo, V. Primeiro ensaio de competição de herbicidas na cultura do milho, na região de Santa Maria (RS). In: Sem. Bras. Herb. Ervas Dan., X, Santa Maria, 1974. Resumos, 64.

5. Crespy, A. Weed control trials in maize. In Compte Rendu de la 7. ${ }^{\circ}$ Conférence du COLUMA (Comité Fraçais de Lutte contre les Mauvaises Heroes). (1973) 499-509. Serv. de la Prot. des végétaux, 280 rue de Fougeres, 3500, Rennes, France (Weed abstract 24(5): 95, 1975).

6. Eschiapati, D. e Dachler, C. Metetilachlor + atrazine, um novo herbicida para a cultura do milho. In: Sem. Bras. Herb. Ervas Dan., XI, Londrina, 1976. Resumos, 44.

7. Hood, R.J. Sutan 6 E - a versatile maize herbicide. Farming in Zambia (1972) 7 (3) 40,46 (Weed Abstract 22(8): 163, 1973).

8. Horowitz, M. Control of grass weeds in corn. In: Proceedings 4th Israeli Weed Control Conférence, Rehovoth, 1970. Tel Aviv, Israel. Weed Science Society of Israel. (1972)53. Voltam: Inst. Agric. Res., Newe ya'ar. (Weed Abstract 22(8): 163, 1973).
9. Salto, E.; Guerra, J.A. e Gainza, A. (Primextra a new herbicide for maize). Primextc _ a, um nuevo herbicida para el main. (Paper given at). Escarda Química en cultivos Herbaceos Extensivos: VIII Jornada de Estudio de la Associación Interprofessional para el Desarollo Agrário, Saragossa, 1976. (1977) 16pp. Ciba Geigy S.A., Barcelona, Spain.

10. Santos, C.A.L. dos e Grassi, N. Aplicação de herbicidas em «pré-plantio» e «pré-emergência» na cultura do milho. O Biológico 35 (11): 255-58, 1969.

11. Santos, C.A.L. dos e Araujo, J.B.M. Estudos relativos à aplicação de herbicidas ha cultura do milho. O Biológico 37(2): 35-38, 1971.

12. Sarpe, N.; Sagarceanu, O. (The use of herbicides mixtures based on atrazine and butylate in irrigated and unirrigated maize crops). Productia Vegetalã - Cereais si Plante Tehnice (1975) 27 (5) 10-16. Inst. Cerc. Cereale Plante Tehnice, Fundulea, Romania. (Weed Abstract 27 (1): 14, 1978).

13. Skalets'Ka, L.I. Fresh fodder yield and quality of maize/soybeans mixture given herbicides. Stepove Zemberobstovo, Resp. Mizhmid. Temat. Nauk. Zb (1975) n. ${ }^{\circ}$ 9, 68-72. From Herbage Abstracts 46,2473. (Weed Abstract 26(11): 345, 1977).

14. Uriarte, J.R. and Garcia, J.G.L. (Chemical control of Cyperus rotundus in maize in El Salvador). Resumenes de Trabajos, 1.a Reunião de Trabajos de la Associación Latinoamericana de Especialistas en las Ciencias Aplicadas a las Malezas (ALAM) 1971, 32 (Weed abstract 21 (3): 191, 1972).

15. Venturella, L.R.C. e Ruckeim, O. Ensaio de herbicidas em milho. In: Sem. Bras. Herb. Ervas Dan., X, Santa Maria, 1974. Resumos, 48.

16. Veselovskii, I.V., Karino, KH.D. (The use of Sutan (butylate) for the chemical weeding of maize. Khimiy v. Sel' skom Goloseevo, Kirv. Ukrainian. SSR. (Weed Abstract 27(9): 312, 1978). 